\title{
Metanálisis de la suplementación con calcio en la prevención de los trastornos hipertensivos del embarazo
}

\author{
Agustín Conde-Agudelo, Ana Cecilia Kafury*; Oscar Arana**
}

RESUMEN OBJETIVO: Evaluar la eficacia de la suplementación con calcio para prevenir los trastornos hipertensivos del embarazo.

MATERIAL Y METODOS: Inicialmente se identificaron las investigaciones clínicas aleatorizadas publicadas después de 1980 acerca de la suplementación con calcio y prevención de los trastornos hipertensivos del embarazo. Los estudios fueron considerados elegibles de acuerdo a su diseño metodológico, análisis estadístico, control de sesgos, etc. A continuación se calculó el Odds ratio (OR) para cada estudio elegido con su respectivo intervalo de confianza del $95 \%$. Finalmente se calculó el Odds ratio típico para el total de los estudios. Cuando el intervalo de confianza del $95 \%$ para el Odds ratio excluyó la unidad, se consideró una p<0.005 (estadísticamente significativo).

RESULTADOS: EI matanálisis de las investigaciones clínicas aleatorizadas mostró que la suplementación con calcio durante el embarazo reduce en forma significativa las incidencias de preeclampsia (OR típico=0.34; IC $95 \%=0.22-0.54)$ y de la hipertensión gestacional (OR típico=0.44; IC 95\%=0.33-0.59).

CONCLUSION: Al presente, la suplementación con calcio durante el embarazo, reduce significativamente la incidencia de los trastornos hipertensivos gestacionales.

SUMMARY OBJECTIVE: To evaluate the effectiveness of calcium supplementation in the prevention of hypertensive pregnancy spells.

MATERIAL AND METHODS: Initially, the clinical recovery investigations published after 1980, about the supplementation with calcium and the prevention of the hypertensive pregnancy spells, were identified. The studies were considered eligible according to their methodological design, statistical analysis, sloped control, etc. Inmediately above, the Odds ratio (OR) were calculated for each study chosen with a $95 \%$ trust interval. Finally the typical Odds ratio was calculated for the total of the studies. When the trust interval of $95 \%$ for the Odds ratio included the unity, a $\mathbf{p}<0.005$ was considered (statistically significant).

RESULTS: The meta-analysis of the clinical recovery investigations showed that the supplementation with calcium during the pregnancy reduced significantly the incidencies of preeclampsia $(\mathrm{OR}$ typic $=0.34 ;$ IC $95 \%=0.22-0.54)$ and of gestational hypertension $(\mathrm{OR}$ typic $=0.44$; IC $95 \%=0.33-0.59)$.

CONCLUSION: At the present time, the supplementation with calcium during the pregnancy reduces significantly the incidency of the gestational hypertensive spells.

\section{Introducción}

Los trastornos hipertensivos del embarazo (THE) son causa primaria de morbimortalidad materna y perinatal especialmente en países en vía de desarrollo (1-4).

En 1980, Belizán y Villar (5) sugirieron la relación entre la ingesta de calcio y el desarrollo de preeclampsia. Desde entonces, varias investigaciones clínicas aleatorizadas han sido realizadas con el fin de evaluar la eficacia de esta intervención en la prevención de los THE. Sin embargo, la gran mayoría de los estudios publicados tienen un tamaño muestral pequeño y diferentes enfoques terapéuticos, haciendo que las conclusiones no sean válidas.

El Metanálisis u Overview es una nueva disciplina que revisa en forma crítica y combina estadísticamente los

\footnotetext{
* Ginecólogo y Obstetra - Investigador y Epidemiólogo Perinatal. Fundación para la Investigación en Reproducción y Medicina Perinatal (INVEST). Cali.

** Director Clínica Rafael Uribe Uribe - ISS. Cali.
}

resultados de trabajos previos. Los propósitos del metanálisis son: 1) Incremento del poder estadístico para los puntos finales primarios y para subgrupos, 2) Resolver incertidumbres cuando los reportes están en desacuerdo, 3) Mejorar los estimativos de la magnitud del efecto y, 4) Responder preguntas no hechas en el inicio de los trabajos individuales.

El objetivo del presente estudio fue revisar la relación entre la ingesta de bajas dosis de aspirina en la prevención de los THE mediante la metodología del metanálisis de estudios existentes.

\section{Materiales y métodos}

Estudios de suplementación con calcio en la prevención de los THE fueron revisados en la literatura médica (idiomas inglés y español). Los procedimientos para identificar tales estudios incluyeron: Búsqueda asistida por computador (MEDLINE), bibliografía de estudios publicados, ODPT (The Oxford Database of Perinatal Trials) y capítulos en 
textos standard sobre hipertensión en el embarazo. Solamente fueron consideradas investigaciones clínicas aleatorizadas (randomizadas). La elegibilidad fue determinada por la lectura de las secciones de materiales y métodos (primariamente) y resultados (secundariamente). Los estudios fueron considerados elegibles si ellos alcanzaron los siguientes criterios:

1) La definición de THE fue establecida claramente. En la discusión de los resultados, el término hipertensión gestacional fue definido como hipertensión (presión arterial sistólica $>90 \mathrm{~mm} \mathrm{Hg}$ ) sin proteinuria después de la semana 20 de gestación o durante el período postparto y el término preeclampsia se reservó para la combinación de hipertensión gestacional y proteinuria.

2) Un grupo control debe haber sido incluido en la investigación del tratamiento y la asignación de la intervención debe haber ocurrido por un método formal de randomización.

3) Los datos numéricos para todas las variables bajo estudio deben haber sido expuestos separadamente.

Los datos fueron extraídos por los dos autores y discutidos conjuntamente. Tradicionalmente, los ensayos individuales son agrupados de acuerdo a la clase de tratamiento, el punto final de interés, $\mathrm{o}$ ambos, son presentados en orden descendente de acuerdo al año de publicación o a la calidad del estudio. Las diferencias agrupadas entre los grupos tratamiento y control son luego presentadas como una sumatoria de los resultados de los ensayos individuales.

El modelo estadístico empleado en el presente metanálisis es el descrito por Mantel y Haenszel (6) que asume efectos idénticos del tratamiento en los estudios (homogeneidad del verdadero efecto del tratamiento) y las varianzas alrededor de cada efecto promedio dependen, primariamente, del tamaño de cada estudio. Los resultados de Mantel y Haenszel son usualmente reportados como un Odds Ratio con varianzas calculadas por el procedimiento de Peto y que fue descrito por Yusuf (7) y Collins (8). El Odds Ratio representa la probabilidad de ocurrencia de un evento en el grupo tratado comparado con la misma probabilidad en el grupo control. Los Odds ratios de cada estudio generan finalmente un "Typical Odds Ratio" que sintetiza la totalidad de la evidencia estudiada. Para los Odds Ratios individuales y "Typical" se calcularon intervalos de confianza del $95 \%$ usando los procedimientos estadísticos ya descritos (8-9). Cuando el intervalo de confianza del $95 \%$ para el Odds Ratio excluye la unidad, se afirma que este estimativo ha alcanzado el valor nominal de $\mathrm{p}<0.05$.

Collins (8) enfatiza que la información provista por un metanálisis, más que asumir que los efectos del tratamiento sean exactamente del mismo tamaño, indica que los efectos reales en los diferentes estudios "apuntan en la misma dirección general".

\section{Resultados}

Ocho estudios evaluaron la asociación entre la suplementación con calcio y la incidencia de THE. De estos estudios dos fueron rechazados por razones que serán discutidas posteriormente. Los restantes 6 estudios evaluaron las dos entidades (hipertensión gestacional y preeclampsia).
En 1985, Kawasaki y Col. (9) realizaron un estudio que mostró una disminución en la incidencia de THE en pacientes que recibieron suplementación en calcio. Se les administró $600 \mathrm{mg}$ de calcio-l-aspartato oral por día $(156 \mathrm{mg}$ de calcio elemental), a 22 embarazadas desde la semana 20 de gestación hasta el parto. 72 mujeres que no recibieron ninguna medicación sirvieron como grupo control. Se realizó la prueba de sensibilidad a la angiotensina II por el método de Gant a las 20, 26, 30 y 36 semanas. Esta mostró una sensibilidad significativamente menor después de la ingesta de calcio, apreciándose la mayor diferencia respecto al grupo control a las 26 y 30 semanas. La incidencia de THE en el grupo que recibió calcio fue $4.5 \%$ (1 paciente) mientras que en el grupo control fue de $21.2 \%$, lo que no presentó una diferencia significativa entre ambos grupos $(\mathrm{p}=0.08)$. En vista de que la dosis utilizada de calcio fue muy baja y que desde el punto de vista metodológico existen muchos elementos de sesgo, tales como la falta de randomización en la asignación del tratamiento, la no utilización de placebo en el grupo control y la falta de enmascaramiento, decidimos no incluirlo en el metanálisis.

Marya y Col. (10) investigaron el efecto de la suplementación con calcio y vitamina D sobre la incidencia de THE. El estudio se realizó en 400 mujeres embarazadas nulíparas hindúes. 200 pacientes fueron asignadas aleatoriamente a recibir calcio (375 mg) y vitamina D (1200 UI) diariamente, desde la semana 20-24 hasta el parto y las restantes 200 recibieron placebo. La incidencia de THE en el grupo calcio fue $6 \%$ y en el grupo placebo $9 \%$ ( $p>0.05$ ). Este estudio fue excluido del metanálisis por la evaluación conjunta de las drogas, las bajas dosis de calcio utilizado y el tamaño muestral no adecuado.

En 1987, Montanaro y Col. (11) realizaron una investigación clínica aleatorizada simple ciego, donde estudiaron el efecto de $2 \mathrm{~g}$ de calcio diario vía oral, administrado a partir de la semana 24 de gestación hasta el parto, en 84 embarazadas. El grupo control se integró con 86 embarazadas. Todas eran sanas y normotensas al inicio del estudio. La incidencia de preeclampsia fue de $10.4 \%$ en el grupo control y $2.3 \%$ en el tratado, mientras que la incidencia de hipertensión gestacional fue de $16.2 \%$ y $4.7 \%$ respectivamente.

López-Jaramillo y Col. (12-13) realizaron dos estudios clínicos en una población de embarazadas residentes en Quito (Ecuador). Los criterios de selección fueron nuliparidad, edad $<25$ años, primer control prenatal antes de la semana 24 de gestación y TA $<120 / 80 \mathrm{~mm} \mathrm{Hg}$ en ese primer control. En el primer estudio participaron $106 \mathrm{pa}-$ cientes que fueron asignadas aleatoriamente a dos grupos. En uno $(\mathrm{N}=55)$, las pacientes recibieron tabletas con $2 \mathrm{~g} /$ día de calcio elemental hasta el día del parto; en el otro $(\mathrm{N}=51)$ recibieron placebo. La incidencia de THE en el grupo calcio fue de $4.1 \%$ y en el grupo placebo $27.9 \%$. No se registraron casos de mortalidad perinatal, bajo peso o partos de pretérmino. El promedio de pesos de los recién nacidos fue mayor en el grupo suplementado con calcio, aunque la diferencia no alcanzó significación estadística. La segunda investigación incluyó 56 nulíparas de iguales características que las anteriores, pero que fueron consideradas en alto riesgo de desarrollar THE por presentar un Roll Over Test (prueba de presión supina) positiva. La incidencia de THE 
en el grupo tratado fue de $14 \%$ mientras que en el grupo placebo fue de $71 \%$. Ambos estudios demostraron un efecto protector del calcio para el desarrollo de THE. La magnitud de los resultados es atribuible a la alta incidencia de THE en esa población seleccionada.

-Villar y Col (14-15) realizó dos investigaciones clínicas aleatorizadas evaluando la utilidad de la suplementación con calcio durante el embarazo en la prevención de los THE y parto pretérmino. En el primer estudio 52 embarazadas nulíparas sanas fueron asignadas en forma aleatoria a recibir $2 \mathrm{~g} /$ día de calcio elemental $(\mathrm{N}=25)$ o placebo $(\mathrm{N}=27)$. La incidencia de THE en el grupo tratado fue $8 \%$ y en grupo control $22.2 \%$, aunque esta diferencia no fue estadísticamente significativa. En 1990 se publicó el segundo estudio el cual tenía una metodología similar al anterior. 90 pacientes recibieron $2 \mathrm{~g}$ diarios de calcio elemental desde la semana 20 hasta el parto mientras 88 constituyeron el grupo control. La incidencia de THE fue de $3.3 \%$ en el grupo tratado y $12.5 \%$ en el grupo placebo $(\mathrm{p}<0.05)$. Además, la incidencia de parto pretérmino fue significativamente menor en el grupo tratado que en el placebo $(7.4 \%$ y $21 \%$ respectivamente).

En 1991, Belizán y Col. (16) realizaron en Rosario (Argentina) una investigación clínica aleatorizada doble ciego con el fin de evaluar el efecto de la suplementación diaria con $2 \mathrm{~g}$ de calcio sobre la incidencia de THE. Los criterios de inclusión fueron: Nuliparidad, embarazo con feto único, edad gestacional $<20$ semanas y ausencia de enfermedad. Se reclutaron 1.194 pacientes que fueron asignadas por randomización a dos grupos para recibir 2 g/día de calcio o placebo. En el análisis final participaron 588 pacientes del grupo placebo y 579 en el grupo de calcio, ya que 27 pacientes $(2.3 \%$ ) se perdieron de la randomización. Los resultados mostraron una incidencia de hipertensión gestacional de $10.7 \%$ en el grupo placebo y $7.2 \%$ en el grupo tratado, diferencia que fue estadísticamente significativa $(\mathrm{p}<0.05)$. Aunque la incidencia de preeclampsia fue menor en el grupo tratado con calcio con respecto al placebo $(2.6 \%$ y $3.9 \%$ ), la diferencia no fue estadísticamente significativa. En 780 pacientes randomizadas se determinó la relación calcio/creatinina y se encontró que el efecto mayor de la suplemantación con calcio en la reducción de los THE fue en el grupo de pacientes que tenía una relación calcio/ creatinina baja. No se registraron efectos colaterales clínicos con estas dosis de calcio. Este estudio es el de mejor diseño por el tamaño muestral calculado correctamente y por el manejo impecable que se le dio al enmascaramiento, seguimiento y prevención en los sesgos.

El metanálisis de la suplementación con calcio durante el embarazo e incidencia de preeclampsia (tabla 1) reúne un total de 1.729 pacientes evaluadas (855 recibieron calcio y 874 placebo). En todos los estudios se aprecia una menor incidencia de preeclampsia en el grupo tratado con calcio con respecto al grupo placebo, aunque en tres de ellos la diferencia no fue estadísticamente significativa. La medida resumen de estos trabajos determinada a través del "typical odds ratio" expresa una reducción significativa de $66 \%$ en el desarrollo de preeclampsia en el grupo que recibió calcio $(\mathrm{OR}=0.34$; IC95\%=0.22-0.54).

En la tabla 2 se aprecia el efecto de la suplementación con calcio en la incidencia de hipertensión gestacional valorado por un metanálisis que incluye los mismos 6 trabajos con el diseño de investigaciones clínicas aleatorizadas. En todos ellos se manifiesta la menor incidencia de hipertensión gestacional en el grupo tratado con ASA respecto al placebo, aunque en dos de ellos no hubo significancia estadística (el valor de 1 se incluyó en los intervalos de confianza). El "typical Odds ratio" de 0.44

Tabla 1

METANALISIS DE LA SUPLEMENTACION CON CALCIO Y PREECLAMPSIA

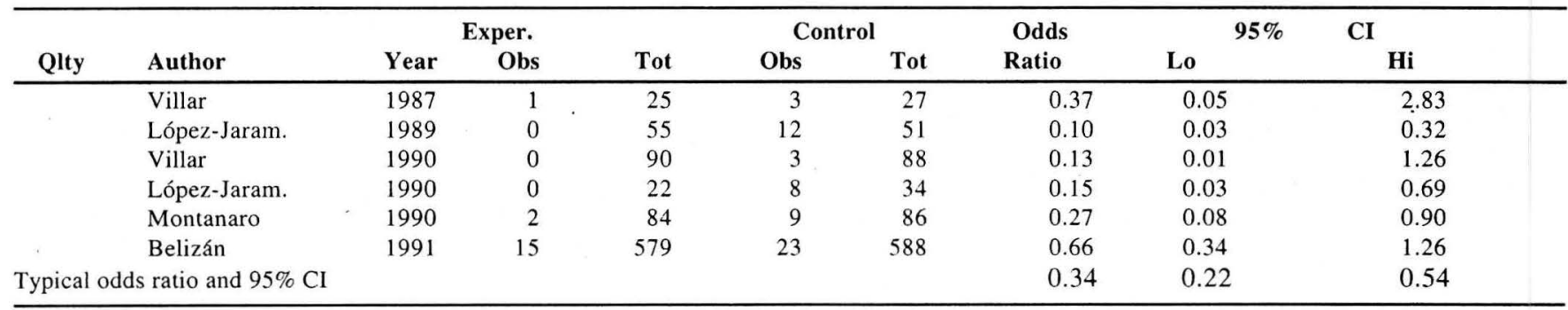

Tabla 2

METANALISIS DE LA ADMINISTRACION DE ASPIRINA E HIPERTENSION GESTACIONAL

\begin{tabular}{|c|c|c|c|c|c|c|c|c|c|}
\hline $\begin{array}{l}\text { Exper. } \\
\text { Qlty }\end{array}$ & $\begin{array}{l}\text { Control } \\
\text { Author }\end{array}$ & $\begin{array}{l}\text { Odds } \\
\text { Year }\end{array}$ & $\begin{array}{l}95 \% \\
\text { Obs }\end{array}$ & $\begin{array}{r}\text { CI } \\
\text { Tot }\end{array}$ & Obs & Tot & Ratio & Lo & Hi \\
\hline & Wallemburg & 1986 & 3 & 23 & 4 & 23 & 0.72 & 0.15 & 3.53 \\
\hline & Schiff & 1989 & 3 & 34 & 4 & 31 & 0.66 & 0.14 & 3.13 \\
\hline & Sibai & 1989 & 0 & 30 & 0 & 10 & 1.00 & 1.00 & 1.00 \\
\hline & Benigni & 1989 & 0 & 17 & 3 & 16 & 0.11 & 0.01 & 1.15 \\
\hline & Uzan & 1990 & 40 & 156 & 25 & 73 & 0.66 & 0.35 & 1.21 \\
\hline & McParland & 1990 & 6 & 36 & 13 & 42 & 0.47 & 0.17 & 1.30 \\
\hline & Davies & 1991 & 3 & 33 & 8 & 34 & 0.35 & 0.10 & 1.28 \\
\hline & Sibai & 1993 & 100 & 1485 & 89 & 1500 & 1.14 & 0.85 & 1.54 \\
\hline & Hauth & 1993 & 19 & 302 & 17 & 302 & 1.13 & 0.57 & 2.21 \\
\hline \multicolumn{7}{|c|}{ Typical odds ratio and $95 \% \mathrm{CI}$} & 0.93 & 0.74 & 1.17 \\
\hline
\end{tabular}


indica una protección del $56 \%$ y es estadísticamente significativa (IC95\% $=0.33-0.59)$.

Las figuras 1 y 2 expresan de manera gráfica los datos de las tablas 1 y 2 .

\section{Discusión}

La relación entre ingesta elevada de calcio y THE surge a partir de observaciones hechas por Belizán y Villar (5) en poblaciones guatemaltecas, donde mujeres embarazadas con factores de riesgo para la aparición de THE, tales como desnutrición, bajo nivel socio-económico y control prenatal insuficiente, muestran una incidencia de THE entre las más bajas del mundo. Se detectó que, a pesar de consumir una dieta inadecuada en la mayoría de sus componentes, el consumo de calcio era alto. Observaciones similares han sido realizadas en Etiopía. Estos hechos llevaron a la realización de varias investigaciones clínicas aleatorizadas

\section{Figura 1}

\section{METANALISIS DE LA SUPLEMENTACION CON \\ CALCIO DURANTE LL EMBARAZO PARA LA PREVENCION DE I A PREECLAMPSIA}

\author{
VILLAR \\ LOPEZ-JARAM \\ VILLAR \\ LOPEZ-JARAM. \\ MONTANARO \\ BEL I ZAN \\ TYPICAL ODDS RATIO
}

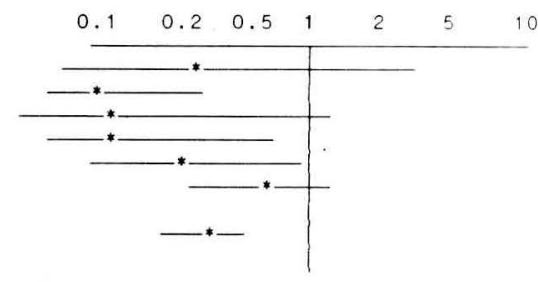

Figura 2

METANALISIS DE LA SUPLEMIENTCION CON

CALCIO DURANTE EL EMBARAZO PARA LA

PREVENCION DE LA HIPERTENSION GESTACIONAL

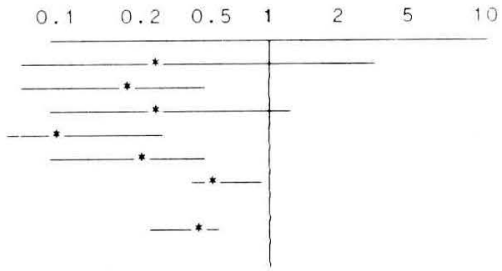

que evaluaron la suplementación con $2 \mathrm{~g}$ /día de calcio y la incidencia de THE.

Aunque todos los estudios citados apuntan hacia la misma dirección del efecto protector de calcio en la incidencia de preeclampsia, la magnitud del efecto varía mucho por el inadecuado tamaño muestral utilizado, a excepción del trabajo de Belizán (16). Sin embargo, llama la atención que en este último trabajo (metodológicamente considerado el mejor) la diferencia no hubiera sido estadísticamente significativa.

Al evaluar la relación entre ingesta de calcio e hipertensión gestacional es también evidente la disminución significativa de este desorden.

Otro resultado interesante que se ha obtenido con la suplementación de calcio es la disminución significativa de parto pretérmino entre las usuarias con respecto al grupo control. No hay evidencia suficiente del efecto del calcio en variables como peso al nacer, mortalidad perinatal o índice de cesáreas.

En ninguno de los trabajos analizados se encontró evidencia de efectos adversos feto-neonatales o maternos. La inquietud que surge tiene que ver con los hipotéticos efectos a largo plazo tales como litiasis materna y osteopatías neonatales. Se tiene conocimiento que, en la actualidad, la gran mayoría de pacientes involucrados en estos estudios están siendo seguidas cuidadosamente para evaluar estas inquietudes.

Persisten sin embargo algunas dudas como el hecho de ser solamente tres los grupos que han evaluado este problema y valdría la pena tener reportes de estudios similares realizados en diversas partes del mundo y así, tener una visión más global. En la actualidad, Levine y Cols. realizan una investigación clínica aleatorizada multicéntrica que involucra alrededor de 3000 pacientes en varias ciudades de los Estados Unidos y que estará concluida durante el presente año. Este estudio muy seguramente dará respuesta a muchos interrogantes y definirá la adopción de la suplementación con calcio como una práctica rutinaria en el control prenatal.

En conclusión:

1) Hasta la fecha, la suplementación con 2 g/día de calcio durante el embarazo disminuye significativamente las incidencias de hipertensión gestacional y preeclampsia.

2) Efectos adversos feto-neonatales y maternos no han sido reportados.

3) La suplementación con calcio disminuye significativamente el parto pretérmino, aunque no ha disminuido otros índices importantes relacionados con la morbimortalidad perinatal.

\section{BIBLIOGRAFIA}

1. Chesley LC. Hypertensive disorders in pregnancy. New York: Appleton-Century-Crofts 1978; 2-3.

2. Kaunitz AM., Hughes JM., Grimes DA., Smith JC., Rochat RW., Kafrissen ME. Causes of maternal mortality in the United States. Obstet. Gynecol. 1985; 65: 605-612.
3. Cunningham FG., MacDonald PC., Gant NF. Hypertensive disorders in pregnancy. In: Cunningham FG, MacDonald PC, Gant NF, eds. Williams'Obstetrics. 18th ed. Norwalk, Connecticut: Appleton \& Lange, 1989; 653.

4. Duley L. Maternal mortality associated with hypertensive disorders of pregnancy in Africa, Asia, Latin America and the Caribbean. Br. J. Obstet. Gynecol. 1992; 99: 547-553. 
5. Belizan JM., Villar J. The relationship between calcium intake and edema-proteinuria and hypertension-gestosis: an hypothesis. Am. J. Clin. Nutr. 1980; 33: 2202-2210.

6. Mantel N., Haenszel W. Statistical aspects of the analysis of data from retrospective studies of disease. J. Natl. Cancer. Inst. 1959; 22: 719748.

7. Yusuf S., Peto R., Lewis J., Collins R., Sleigh P. Beta blockade during and after myocardial infarction: an overview of the randomized trials. Prog. Cardiovasc. Dis. 1985; 27: 335-371.

8. Collins R., Scrimgeour A., Yusuf S., Peto R. Reduction in fatal pulmonary embolism and venous thrombosis by perioperative administration of subcutaneous heparin: overview of results of randomized trials in general, orthopedic, and urology surgery. $\mathrm{N}$. Engl. J. Med. 1988; 318: 1162-1173.

9. Kawasaki N., Matsui K., Ito M. et al. Effect of calcium supplementation on the vascular sensitivity to angiotensin II in pregnant women. Am. J. Obstet. Gynecol. 1987; 153: 576-579.

10. Marya RK., Rathee S., Manrow M. Effect of calcium with vitamin D supplementation on toxaemia of pregnancy. Gynecol. Obstet. Invest. 1987; 24: 38-42.
11. Montanaro D., Boscutti G., Antonucci F. et al. Prevention of pregnancyinduced hypertension (PIH) and preeclampsia (PE) by oral calcium supplementation. Presented at the 10th International Congress of Nephrology, London, july 26-31, 1987. Abstract.

12. Lopez-Jaramillo P., Narvaez M., Weigel RM., Yepez R. Calcium supplementation reduces the risk of pregnancy-induced hypertension in an Andes population. J. Obstet. Gynecol. 1989; 19: 648-655.

13. Lopez-Jaramillo P., Narvaez M., Felix C., Lopez A. Dietary calcium supplementation and prevention of pregnancy hypertension. Lancet 1990; 335: 293.

14. Villar J., Repke J., Belizan JM., Pareja G. Calcium supplementation reduces blood pressure during pregnancy: results of a randomized controlled clinical trial. Obstet. Gynecol. 1987; 70: 317-322.

15. Villar J., Repke JT. Calcium supplementation during pregnancy may reduce preterm delivery in high-risk populations. Am. J. obstet. Gynecol. 1990; 163: 1124-1131.

16. Belizan JM., Villar J., Gonzalez L., Campodonico L., Bergel E. Calcium supplementation to prevent hypertensive disorders of pregnancy. N. Engl. J. Med. 1991; 325: 1399-1405. 